\title{
Nonlinear contractive and nonlinear compatible type mappings in Menger probabilistic metric spaces
}

Yong Kun Tang, Shih-sen Chang ${ }^{*}$ and Lin Wang

Dedicated to Professor Shih-sen Chang on the occasion of his 80th birthday

*Correspondence:

changss2013@163.com

College of Statistics and

Mathematics, Yunnan University of

Finance and Economics, Kunming,

Yunnan 650221, China

\begin{abstract}
In this paper some new fixed point theorems for nonlinear contractive type and nonlinear compatible type mapping in complete Menger probabilistic metric spaces are proved.

MSC: 54E40; 54E35; 54H25

Keywords: distribution function; probabilistic metric spaces; completeness; nonlinear contraction; compatible mapping; fixed point theorem
\end{abstract}

\section{Introduction and preliminaries}

K Menger introduced the notion of a probabilistic metric space in 1942. Since then the theory of probabilistic metric spaces has developed in many directions $[1,2]$. The idea of $\mathrm{K}$ Menger was to use distribution functions instead of non-negative real numbers as values of the metric. The notion of a probabilistic metric space corresponds to situations when we do not know exactly the distance between two points, but we know probabilities of possible values of this distance. A probabilistic generalization of metric spaces appears to be of interest in the investigation of physical quantities and physiological thresholds. It is also of fundamental importance in probabilistic functional analysis.

The purpose of this paper is to prove some existence theorems of fixed points for nonlinear contractive type and nonlinear compatible type mapping in complete Menger probabilistic metric spaces. In the sequel, we shall adopt the usual terminology, notation and conventions of the theory of probabilistic metric, as in [1-6].

Throughout this paper, let $\mathbb{R}$ be the set of all real numbers and $\mathbb{R}^{+}$be the set of all nonnegative real numbers. A mapping $\mathcal{F}: \mathbb{R} \rightarrow \mathbb{R}^{+}$is called a distribution function (briefly, d.f.), if it is left-continuous and non-decreasing with

$$
\inf _{R} \mathcal{F}=0, \quad \sup _{R} \mathcal{F}=1
$$

In the sequel, we denote by $\Delta^{+}$the set of all distribution functions on $\mathbb{R}$. The space $\Delta^{+}$ is partially ordered by the usual point-wise ordering of functions, i.e., $\mathcal{F} \leq \mathcal{G}$ if and only if

\section{Springer}

๑2014 Tang et al.; licensee Springer. This is an Open Access article distributed under the terms of the Creative Commons Attribution License (http://creativecommons.org/licenses/by/2.0), which permits unrestricted use, distribution, and reproduction in any medium, provided the original work is properly cited. 
$\mathcal{F}(t) \leq \mathcal{G}(t)$ for all $t \in \mathbb{R}$. The maximal element for $\Delta^{+}$in this order is the d.f. given by

$$
H(t)= \begin{cases}0, & t \leq 0 \\ 1, & t>0\end{cases}
$$

Definition 1.1 $[1,2]$ A mapping $T:[0,1] \times[0,1] \rightarrow[0,1]$ is called a continuous $t$-norm, if $T$ satisfies the following conditions:

(a) $T$ is commutative and associative;

(b) $T$ is continuous;

(c) $T(a, 1)=a$ for all $a \in[0,1]$;

(d) $T(a, b) \leq T(c, d)$ whenever $a \leq c, b \leq d$, and $a, b, c, d \in[0,1]$.

Two typical examples of continuous $t$-norm are $T(a, b)=a b$ and $T(a, b)=\min \{a, b\}$.

Now $t$-norms are recursively defined by $T^{1}=T$ and

$$
T^{n}\left(x_{1}, x_{2}, \ldots, x_{n+1}\right)=T\left(T^{n-1}\left(x_{1}, x_{2}, \ldots, x_{n}\right), x_{n+1}\right)
$$

for all $n \geq 2$ and $x_{i} \in[0,1]$, for all $i=1,2, \ldots, n+1$.

Definition 1.2 A Menger Probabilistic Metric space (briefly, Menger PM-space) is a triple $(X, \mathcal{F}, T)$, where $X$ is a non-empty set, $T$ is a continuous $t$-norm, and $\mathcal{F}$ is a mapping from $X \times X$ into $\Delta^{+}$satisfying the following conditions: for all $x, y, z \in X$ (in the sequel, we use $F_{x, y}$ to denote $\left.\mathcal{F}(x, y)\right)$ :

(PM1) $F_{x, y}(t)=H(t), \forall t>0$, if and only if $x=y$;

(PM2) $F_{x, y}(t)=F_{y, x}(t)$;

(PM3) $F_{x, z}(t+s) \geq T\left(F_{x, y}(t), F_{y, z}(s)\right), \forall x, y, z \in X$ and $t, s \geq 0$.

Definition 1.3 Let $(X, \mathcal{F}, T)$ be a Menger PM-space.

(1) A sequence $\left\{x_{n}\right\}$ in $X$ is said to be convergent to $x \in X$ if, for every $\epsilon>0$ and $\lambda>0$, there exists a positive integer $N$ such that $F_{x_{n}, x}(\epsilon)>1-\lambda$ whenever $n \geq N$.

(2) A sequence $\left\{x_{n}\right\}$ in $X$ is called a Cauchy sequence if, for every $\epsilon>0$ and $\lambda>0$, there exists a positive integer $N$ such that $F_{x_{n}, x_{m}}(\epsilon)>1-\lambda$ whenever $n, m \geq N$.

(3) A Menger PM-space $(X, \mathcal{F}, T)$ is said to be complete if and only if every Cauchy sequence in $X$ is convergent to a point in $X$.

Definition 1.4 Let $(X, \mathcal{F}, T)$ be a Menger PM space, $p \in X$ be a given point.

(1) For any given $\epsilon>0$ and $\lambda>0$ the set

$$
N p(\epsilon, \lambda)=\left\{q \in X: F_{p, q}(\epsilon)>1-\lambda\right\}
$$

is called the strong $(\epsilon, \lambda)$-neighborhood of $p$.

(2) The strong neighborhood system for $X$ is the union $\bigcup_{p \in X} M_{p}$, where $M_{p}=\{N p(\epsilon, \lambda), \epsilon>0, \lambda>0\}$.

Remark 1.5 It should be pointed out that the strong neighborhood system determines a Hausdorff topology $\sigma$ on $X[1,2]$. 
Definition 1.6 [2] Let $X$ be a non-empty set, $\left\{d_{\alpha}: \alpha \in(0,1)\right\}$ be a family of mappings from $X \times X$ into $\mathbb{R}^{+}$. The ordered pair $\left(X, d_{\alpha}: \alpha \in(0,1)\right)$ is called a generating space of quasimetrics family, and $\left\{d_{\alpha}: \alpha \in(0,1)\right\}$ is called the family of quasi-metrics on $X$ if, it satisfies the following conditions:

(QM-1) $d_{\alpha}(x, y)=0$ for all $\alpha \in(0,1)$, if and only if $x=y$;

$(\mathrm{QM}-2) d_{\alpha}(x, y)=d_{\alpha}(y, x)$ for all $\alpha \in(0,1)$ and $x, y \in X$;

(QM-3) for any given $\alpha \in(0,1)$, there exists $\mu \in(0, \alpha]$ such that

$$
d_{\alpha}(x, y) \leq d_{\mu}(x, z)+d_{\mu}(z, y), \quad \forall x, y, z \in X
$$

(QM-4) for any give $x, y \in X$, the function $\alpha \mapsto d_{\alpha}(x, y)$ is nonincreasing and left-continuous.

Lemma 1.7 Let $(X, \mathcal{F}, T)$ be a Menger PM-space with a $t$-norm $T$ satisfying the following conditions:

$$
\sup _{t<1} T(t, t)=1
$$

For any given $\lambda \in(0,1)$, define a mapping $E_{\lambda, F}(x, y): X \times X \rightarrow R^{+}$as follows:

$$
E_{\lambda, F}(x, y)=\inf \left\{t>0: F_{x, y}(t)>1-\lambda\right\},
$$

then

(1) $\left(E_{\lambda, F}: \lambda \in(0,1)\right)$ is a family of quasi-metrics on $X$ and $\left(X, E_{\lambda, F}: \lambda \in(0,1)\right)$ is a generating space of the quasi-metrics family $\left\{E_{\lambda, F}: \lambda \in(0,1)\right\}$;

(2) the topology induced by quasi-metric family $\left\{E_{\lambda, F}: \lambda \in(0,1)\right\}$ on $X$ coincides with the $(\epsilon, \lambda)$-topology on $X$.

Proof (1) From Definition 1.6, it is easy to see that the family $\left\{E_{\lambda, F}: \lambda \in(0,1)\right\}$ satisfies the conditions (QM-1) and (QM-2).

Next we prove that $E_{\lambda, F}$ is left-continuous in $\lambda \in(0,1)$. In fact, for any given $\lambda_{1} \in(0,1)$ and $\epsilon>0$, by the definition of $E_{\lambda, F}$, there exists $t_{1}>0$ such that $t_{1}<E_{\lambda_{1}, F}(x, y)+\epsilon$ and $F_{x, y}\left(t_{1}\right)>1-\lambda_{1}$. Letting $\delta=F_{x, y}\left(t_{1}\right)-\left(1-\lambda_{1}\right)>0$ and $\lambda \in\left(\lambda_{1}-\delta, \lambda_{1}\right)$, we have

$$
1-\lambda_{1}<1-\lambda<1-\left(\lambda_{1}-\delta\right)=F_{x, y}\left(t_{1}\right) .
$$

This implies that

$$
t_{1} \in\left\{t>0: F_{x, y}(t)>1-\lambda\right\}
$$

Hence we have

$$
E_{\lambda_{1}, F}(x, y) \leq E_{\lambda, F}(x, y)(t)=\inf \left\{t>0: F_{x, y}>1-\lambda\right\} \leq t_{1}<E_{\lambda_{1}, F}(x, y)+\epsilon,
$$

which shows $\lambda \mapsto E_{\lambda, F}$ is left-continuous.

Now we prove that, for any given $(x, y) \in X \times X, E_{\lambda, F}$ is nonincreasing in $\lambda \in(0,1)$. 
In fact, for any $\lambda_{1}, \lambda_{2} \in(0,1)$ with $\lambda_{1}<\lambda_{2}$, we have

$$
\left\{t>0: F_{x, y}(t)>1-\lambda_{1}\right\} \subset\left\{t>0: F_{x, y}(t)>1-\lambda_{2}\right\}
$$

Hence

$$
\begin{aligned}
E_{\lambda_{2}, F}(x, y) & =\inf \left\{t>0: F_{x, y}(t)>1-\lambda_{2}\right\} \\
& \leq E_{\lambda_{1}, F}(x, y)=\inf \left\{t>0: F_{x, y}(t)>1-\lambda_{1}\right\}
\end{aligned}
$$

Condition (QM-4) is proved.

Finally, we prove that $\left\{E_{\lambda, F}: \lambda \in(0,1)\right\}$ also satisfies condition (QM-3).

In fact, for any given $x, y \in X$, and $\lambda \in(0,1)$, by condition $(1.1)$, there exists $\mu \in(0, \lambda]$ such that

$$
T^{n}(1-\mu, 1-\mu, \ldots, 1-\mu)>1-\lambda
$$

Letting $E_{\mu, F}\left(x, x_{1}\right)=\delta_{1}, E_{\mu, F}\left(x_{1}, x_{2}\right)=\delta_{2}, \ldots, E_{\mu, F}\left(x_{n}, y\right)=\delta_{n+1}$, from (1.2) for any $\epsilon>0$, we have

$$
F_{x, x_{1}}\left(\delta_{1}+\epsilon\right)>1-\mu, \quad F_{x_{1}, x_{2}}\left(\delta_{2}+\epsilon\right)>1-\mu, \quad \ldots, \quad F_{x_{n}, y}\left(\delta_{n+1}+\epsilon\right)>1-\mu
$$

and so we have

$$
\begin{aligned}
F_{x, y}\left(\delta_{1}+\delta_{2}+\cdots+\delta_{n+1}+(n+1) \epsilon\right) & \geq T^{n}\left(F_{x, x_{1}}\left(\delta_{1}+\epsilon\right), F_{x_{1}, x_{2}}\left(\delta_{2}+\epsilon\right), \ldots, F_{x_{n}, y}\left(\delta_{n+1}+\epsilon\right)\right) \\
& \geq T^{n}(1-\mu, 1-\mu, \ldots, 1-\mu)>1-\lambda .
\end{aligned}
$$

This implies that

$$
E_{\lambda, F}(x, y)=\inf \left\{t>0: F_{x, y}(t)>1-\lambda\right\} \leq \delta_{1}+\delta_{2}+\cdots+\delta_{n+1}+(n+1) \epsilon .
$$

By the arbitrariness of $\epsilon>0$, we have

$$
E_{\lambda, F}(x, y) \leq E_{\mu, F}\left(x, x_{1}\right)+E_{\mu, F}\left(x_{1}, x_{2}\right)+\cdots+E_{\mu, F}\left(x_{n}, y\right)
$$

for any $x, y, x_{1}, x_{2}, \ldots, x_{n} \in X$. Especially, if $n=1$, then condition (QM-3) is proved. The conclusion (1) is proved.

Now we prove the conclusion (2).

For the purpose, it is sufficient to prove that, for any given $\epsilon>0$ and $\lambda \in(0,1)$,

$$
E_{\lambda, F}(x, y)<\epsilon \quad \Leftrightarrow \quad F_{x, y}(\epsilon)>1-\lambda
$$

In fact, if $E_{\lambda, F}(x, y)<\epsilon$, then from (1.2) we have $F_{x, y}(\epsilon)>1-\lambda$. Conversely, if $F_{x, y}(\epsilon)>$ $1-\lambda$, since $F_{x, y}$ is a left-continuous distribution function, there exists a $\mu>0$ such that $F_{x, y}(\epsilon-\mu)>1-\lambda$, and so $E_{\lambda, F}(x, y) \leq \epsilon-\mu<\epsilon$.

This completes the proof of Lemma 1.7. 
Remark 1.8 From Lemma 1.7, it is easy to see that a sequence $\left\{x_{n}\right\}$ in a Menger PM-space $(X, \mathcal{F}, T)$ is convergent in the $(\epsilon, \lambda)$-topology $\sigma$, if and only if $E_{\lambda, F}\left(x_{n}, x\right) \rightarrow 0, \forall \lambda \in(0,1)$. Also a sequence $\left\{x_{n}\right\}$ in a Menger PM-space $(X, \mathcal{F}, T)$ is a Cauchy sequence in the $(\epsilon, \lambda)$ topology, if and only if $E_{\lambda, F}\left(x_{n}, x_{m}\right) \rightarrow 0 \forall \lambda \in(0,1)$ (as $\left.n, m \rightarrow \infty\right)$.

Definition 1.9 A function $\phi:[0, \infty) \rightarrow[0, \infty)$ is said to satisfy condition $(\Phi)$, if it is nondecreasing and $\sum_{n=1}^{\infty} \phi^{n}(t)<\infty$ for all $t>0$, where $\phi^{n}(t)$ denotes the $n$th iterate function of $\phi(t)$.

Remark 1.10 [7, 8] If $\phi:[0, \infty) \rightarrow[0, \infty)$ satisfies condition $(\Phi)$, then $\phi(t)<t, \forall t>0$. If $t \leq \phi(t)$, then $t=0$.

Lemma 1.11 [9] Let $(X, \mathcal{F}, T)$ be a Menger PM-space. Suppose that the function $\phi$ : $[0, \infty) \rightarrow[0, \infty)$ is onto and strictly increasing. Then

$$
\inf \left\{\phi^{n}(t)>0, F_{x, y}(t)>1-\lambda\right\} \leq \phi^{n}\left(\inf \left\{t>0: F_{x, y}(t)>1-\lambda\right\}\right),
$$

for any $x, y \in X, \lambda \in(0,1)$ and $n \geq 1$.

Lemma 1.12 Let $(X, \mathcal{F}, T)$ be a Menger PM-space with a t-norm $T$ satisfying condition (1.1) and $\left\{x_{n}\right\}$ be a sequence in $X$ such that

$$
F_{x_{n}, x_{n+1}}\left(\phi^{n}(t)\right) \geq F_{x_{0}, x_{1}}(t), \quad \forall t>0, n \geq 1,
$$

where $\phi:[0, \infty) \rightarrow[0, \infty)$ is onto, strictly increasing, and satisfies condition $(\Phi)$. If

$$
E_{F}\left(x_{0}, x_{1}\right):=\sup _{\lambda \in(0,1)}\left\{E_{\lambda, F}\left(x_{0}, x_{1}\right)\right\}<\infty,
$$

then $\left\{x_{n}\right\}$ is a Cauchy sequence in $X$.

Proof For any $\lambda \in(0,1)$, it follows from Lemma 1.11 and condition (1.4) that

$$
\begin{aligned}
E_{\lambda, F}\left(x_{n}, x_{n+1}\right) & =\inf \left\{t>0, F_{x_{n}, x_{n+1}}(t)>1-\lambda\right\} \\
& =\inf \left\{\phi^{n}\left(\phi^{n}\right)^{-1}(t)>0, F_{x_{n}, x_{n+1}}\left(\phi^{n}\left(\phi^{n}\right)^{-1}(t)\right)>1-\lambda\right\} \\
& \leq \inf \left\{\phi^{n}\left(\phi^{n}\right)^{-1}(t)>0, F_{x_{0}, x_{1}}\left(\phi^{n}\right)^{-1}(t)>1-\lambda\right\} \\
& \leq \phi^{n}\left(\inf \left\{\left(\phi^{n}\right)^{-1}(t)>0, F_{x_{0}, x_{1}}\left(\phi^{n}\right)^{-1}(t)>1-\lambda\right\}\right. \\
& =\phi^{n}\left(\inf \left\{t>0, F_{x_{0}, x_{1}}(t)>1-\lambda\right\}\right) \\
& =\phi^{n}\left(E_{\lambda, F}\left(x_{0}, x_{1}\right)\right) \\
& \leq \phi^{n}\left(E_{F}\left(x_{0}, x_{1}\right)\right) .
\end{aligned}
$$


For any given positive integers $m, n, m>n$, and for given $\lambda \in(0,1)$, it follows from (1.5) and Lemma 1.7 that there exists a $\mu \in(0, \lambda]$ such that

$$
\begin{aligned}
E_{\lambda, F}\left(x_{n}, x_{m}\right) & \leq E_{\mu, F}\left(x_{n}, x_{n+1}\right)+E_{\mu, F}\left(x_{n+1}, x_{n+2}\right)+\cdots+E_{\mu, F}\left(x_{m-1}, x_{m}\right) \\
& \leq \sum_{j=n}^{m-1} \phi^{j}\left(E_{F}\left(x_{0}, x_{1}\right)\right) \rightarrow 0 \quad(\text { as } n, m \rightarrow \infty) .
\end{aligned}
$$

By Lemma 1.7, $\left\{x_{n}\right\}$ is a Cauchy sequence in $X$.

This completes the proof of Lemma 1.12.

\section{Fixed point theorems of nonlinear contraction type mappings in Menger \\ PM-spaces}

Theorem 2.1 Let $(X, \mathcal{F}, T)$ be a complete Menger PM-space, $\left\{A_{i}, i=1,2, \ldots\right\}$ be a sequence of mappings from $X$ into itself such that, for any two mappings $A_{i}, A_{j}, i \neq j$ and for any $x, y \in X$ and $t>0$,

$$
F_{A_{i} x, A_{j} y}(\phi(t)) \geq \min \left\{F_{x, y}(t), F_{x, A_{i} x}(t), F_{y, A_{j y}}(t)\right\},
$$

where $\phi:[0, \infty) \rightarrow[0, \infty)$ is onto, strictly increasing, and satisfies condition $(\Phi)$. If there exists $x_{0} \in X$ such that

$$
E_{F}\left(x_{0}, A_{1} x_{0}\right):=\sup _{\lambda \in(0,1)} E_{\lambda, F}\left(x_{0}, A_{1} x_{0}\right)<\infty
$$

Then $\left\{A_{i}\right\}$ has a unique common fixed point $x^{*}$ in $X$, and the sequence $\left\{x_{n}\right\}$ defined by

$$
x_{n}=A_{n} x_{n-1}, \quad \forall n \geq 1,
$$

converges to $x^{*}$ in the $(\epsilon, \lambda)$-topology of $X$.

Proof It follows from (2.1) and (2.3) that

$$
\begin{aligned}
F_{x_{1}, x_{2}}(\phi(t)) & =F_{A_{1} x_{0}, A_{2} x_{1}}(\phi(t)) \\
& \geq \min \left\{F_{x_{0}, x_{1}}(t), F_{x_{0}, x_{1}}(t), F_{x_{1}, x_{2}}(t)\right\} \\
& =\min \left\{F_{x_{0}, x_{1}}(t), F_{x_{1}, x_{2}}(t)\right\}, \quad t>0 .
\end{aligned}
$$

If $F_{x_{1}, x_{2}}(t)<F_{x_{0}, x_{1}}(t)$, then from $(2.4)$

$$
F_{x_{1}, x_{2}}(\phi(t)) \geq F_{x_{1}, x_{2}}(t), \quad t>0 .
$$

By induction, we can prove that, for any positive integer $n$,

$$
F_{x_{1}, x_{2}}\left(\phi^{n}(t)\right) \geq F_{x_{1}, x_{2}}(t), \quad t>0 .
$$

Let $n \rightarrow \infty$, we have $F_{x_{1}, x_{2}}(t)=0$ for all $t>0$. This contradicts that $F_{x_{1}, x_{2}}(t)$ is a distribution function. Hence we have

$$
F_{x_{1}, x_{2}}(\phi(t)) \geq F_{x_{0}, x_{1}}(t), \quad t>0 .
$$


Similarly, we have

$$
\begin{aligned}
F_{x_{2}, x_{3}}\left(\phi^{2}(t)\right) & =F_{A_{2} x_{1}, A_{3} x_{2}}\left(\phi^{2}(t)\right) \\
& \geq \min \left\{F_{x_{1}, x_{2}}(\phi(t)), F_{x_{1}, x_{2}}(\phi(t)), F_{x_{2}, x_{3}}(\phi(t))\right\} \\
& =F_{x_{1}, x_{2}}(\phi(t)) \\
& \geq F_{x_{0}, x_{1}}(t), \quad t>0 .
\end{aligned}
$$

By induction, we can prove that

$$
F_{x_{n}, x_{n+1}}\left(\phi^{n}(t)\right) \geq F_{x_{0}, x_{1}}(t), \quad \forall n \geq 1, t>0 .
$$

It follows from condition (2.2) and Lemma 1.12 that $\left\{x_{n}\right\}$ is a Cauchy sequence in $X$. Since $X$ is complete, there exists a point $x^{*} \in X$ such that $x_{n} \rightarrow x^{*}$.

Next we prove that $x^{*}$ is the unique common fixed point of $\left\{A_{i}\right\}$. In fact, for any given positive integers $i, n, n>i$ and for any $\lambda \in(0,1)$, by Lemma 1.11, we have

$$
\begin{aligned}
E_{\lambda, F}\left(x_{n}, A_{i} x^{*}\right)= & \inf \left\{t>0, F_{A_{n} x_{n-1}, A_{i} x^{*}}(t)>1-\lambda\right\} \\
\leq & \inf \left\{t>0, \min \left\{F_{x_{n-1}, x^{*}}\left(\phi^{-1}(t)\right), F_{x_{n-1}, x_{n}}\left(\phi^{-1}(t)\right),\right.\right. \\
& \left.\left.F_{x^{*}, A_{i}\left(x^{*}\right)}\left(\phi^{-1}(t)\right)\right\}>1-\lambda\right\} \\
= & \inf \left\{\phi\left(\phi^{-1}\right)(t)>0, \min \left\{F_{x_{n-1}, x^{*}}\left(\phi^{-1}(t)\right), F_{x_{n-1}, x_{n}}\left(\phi^{-1}(t)\right),\right.\right. \\
& \left.\left.F_{x^{*}, A_{i}\left(x^{*}\right)}\left(\phi^{-1}(t)\right)\right\}>1-\lambda\right\} \\
\leq & \phi\left(\inf \left\{t>0, \min \left\{F_{x_{n-1}, x^{*}}(t), F_{x_{n-1}, x_{n}}(t), F_{x^{*}, A_{i}\left(x^{*}\right)}(t)\right\}>1-\lambda\right\}\right) \\
\leq & \phi\left(\max \left\{E_{\lambda, F}\left(x_{n-1}, x^{*}\right), E_{\lambda, F}\left(x_{n-1}, x_{n}\right), E_{\lambda, F}\left(x^{*}, A_{i} x^{*}\right)\right\}\right) .
\end{aligned}
$$

By virtue of the continuity of $\phi$, we have

$$
\begin{aligned}
E_{\lambda, F}\left(x^{*}, A_{i} x^{*}\right) & =\lim _{n \rightarrow \infty} E_{\lambda, F}\left(x_{n}, A_{i} x^{*}\right) \\
& \leq \lim _{n \rightarrow \infty} \phi\left(\max \left\{E_{\lambda, F}\left(x_{n-1}, x^{*}\right), E_{\lambda, F}\left(x_{n-1}, x_{n}\right), E_{\lambda, F}\left(x^{*}, A_{i} x^{*}\right)\right\}\right) \\
& =\phi\left(E_{\lambda, F}\left(x^{*}, A_{i} x^{*}\right)\right) .
\end{aligned}
$$

From Remark 1.10, it follows that $E_{\lambda, F}\left(x^{*}, A_{i} x^{*}\right)=0$, i.e., $x^{*}=A_{i} x^{*}, \forall i \geq 1$.

Next we prove that $x^{*}$ is the unique common fixed point of $\left\{A_{i}\right\}$ in $X$. In fact, if $y \in X$ is also a common fixed point of $\left\{A_{i}\right\}$, then, for any $\lambda \in(0,1)$ and any $i, j, i \neq j$,

$$
\begin{aligned}
E_{\lambda, F}\left(x^{*}, y\right) & =E_{\lambda, F}\left(A_{i} x^{*}, A_{j} y\right) \\
& =\inf \left\{t>0, F_{A_{i} x^{*}, A_{j} y}(t)>1-\lambda\right\} \\
& \leq \inf \left\{t>0, \min \left\{F_{x^{*}, y}\left(\phi^{-1}(t)\right), F_{x^{*}, x^{*}}\left(\phi^{-1}(t)\right), F_{y, y}\left(\phi^{-1}(t)\right)\right\}>1-\lambda\right\} \\
& =\inf \left\{\phi\left(\phi^{-1}\right)(t)>0, F_{x^{*}, y}\left(\phi^{-1}(t)\right)>1-\lambda\right\} \\
& \leq \phi\left(\inf \left\{(\phi)^{-1}(t)>0, F_{x^{*}, y}\left(\phi^{-1}(t)\right)>1-\lambda\right\}\right)
\end{aligned}
$$




$$
\begin{aligned}
& =\phi\left(\inf \left\{t>0, F_{x^{*}, y}(t)>1-\lambda\right\}\right) \\
& =\phi\left(E_{\lambda, F}\left(x^{*}, y\right)\right),
\end{aligned}
$$

i.e., $E_{\lambda, F}\left(x^{*}, y\right)=0$ by Remark 1.10 . And so $x^{*}=y$.

This completes the proof of Theorem 2.1.

\section{Fixed point theorems for nonlinear compatible type mappings in PM-spaces}

Definition 3.1 Let $(X, \mathcal{F}, T)$ be a Menger PM-space, and let $f$ and $S$ be two mappings from $X$ into itself. $f$ and $S$ are called compatible if $F_{S f x_{n} f S x_{n}}(t) \rightarrow H(t), \forall t>0$, whenever $\left\{x_{n}\right\}$ is a sequence in $X$ such that $\left\{x_{n}\right\}$ and $\left\{S x_{n}\right\}$ converge in the $(\epsilon, \lambda)$-topology to some $x \in X$ as $n \rightarrow \infty$.

Remark 3.2 It should be point out that the concept of compatible mappings was introduced by Jungck [10] in metric space. The concept of compatible mappings introduced here is a generalization of Jungck [10] and Singh et al. [11].

Theorem 3.3 Let $(X, \mathcal{F}, T)$ be a complete Menger PM-space, $f, g, S, G: X \rightarrow X$ be mappings satisfying the following conditions:

(i) $S(X) \subset g(X), G(X) \subset f(X)$;

(ii) $F_{S x, T y}(\phi(t)) \geq \min \left\{F_{f(x), g(y)}(t), F_{f(x), S(x)}(t), F_{g(y), G(y)}(t)\right\}$

for all $x, y \in X, t>0$, where the function $\phi:[0, \infty) \rightarrow[0, \infty)$ is onto strictly increasing and satisfies condition $(\Phi)$. If either $f$ or $g$ is continuous and the pairs $S, f$ and $G, g$ both are compatible, and if there exists an $x_{0} \in X$ such that

$$
E_{F}\left(z_{0}, z_{1}\right):=\sup _{\lambda \in(0,1)}\left(E_{\lambda, F}\left(z_{0}\right), z_{1}\right)<\infty
$$

where $z_{0}=S\left(x_{0}\right), z_{1}=G\left(x_{1}\right)$ and $g\left(x_{1}\right)=S\left(x_{0}\right)$, then $S, G, f, g$ have a unique common fixed point $z$ in $X$.

Proof By condition (i), there exists $x_{1} \in X$ such that $S x_{0}=g\left(x_{1}\right)=z_{0}$. For $x_{1}$, there exists $x_{2} \in X$ such that $G\left(x_{1}\right)=f\left(x_{2}\right)=z_{1}$. Inductively, we can construct sequences $\left\{x_{n}\right\}$ and $\left\{z_{n}\right\}$ as follows:

$$
\left\{\begin{array}{l}
g\left(x_{2 n+1}\right)=S\left(x_{2 n}\right)=z_{2 n}, \\
f\left(x_{2 n+2}\right)=G\left(x_{2 n+1}\right)=z_{2 n+1},
\end{array} \quad \forall n \geq 0 .\right.
$$

It follows from condition (ii) that, for any $t>0$,

$$
\begin{aligned}
F_{z_{2 n}, z_{2 n+1}}(\phi(t)) & =F_{S\left(x_{2 n}\right), G\left(x_{2 n+1}\right)}(\phi(t)) \\
& \geq \min \left\{F_{f\left(x_{2 n}\right), g\left(x_{2 n+1}\right)}(t), F_{f\left(x_{2 n}\right), S x_{2 n}}(t), F_{g\left(x_{2 n+1}\right), G\left(x_{2 n+1}\right)}(t)\right\} \\
& =\min \left\{F_{z_{2 n-1}, z_{2 n}}(t), F_{z_{2 n}, z_{2 n+1}}(t)\right\} .
\end{aligned}
$$

If $F_{z_{2 n-1}, z_{2 n}}(t) \geq F_{z_{2 n}, z_{2 n+1}}(t)$, then from (3.3), $F_{z_{2 n}, z_{2 n+1}}(\phi(t)) \geq F_{z_{2 n}, z_{2 n+1}}(t), \forall t>0$, and so

$$
F_{z_{2 n}, z_{2 n+1}}\left(\phi^{m}(t)\right) \geq F_{z_{2 n}, z_{2 n+1}}(t), \quad \forall t>0 \text { and } m \geq 1 .
$$


Since $\phi$ satisfies condition $(\Phi)$, we have

$$
0=\lim _{m \rightarrow \infty} F_{z_{2 n}, z_{2 n+1}}\left(\phi^{m}(t)\right) \geq F_{z_{2 n}, z_{2 n+1}}(t), \quad \forall t>0 .
$$

This contradicts that $F_{z_{2 n}, z_{2 n+1}}(t)$ is a distribution function. Therefore

$$
F_{z_{2 n}, z_{2 n+1}}(\phi(t)) \geq F_{z_{2 n-1}, z_{2 n}}(t), \quad \forall t>0 .
$$

Similarly we can prove that

$$
F_{z_{2 n+1}, z_{2 n+2}}(\phi(t)) \geq F_{z_{2 n}, z_{2 n+1}}(t), \quad \forall t>0 .
$$

These show that, for any positive integer $m \geq 1$, we have

$$
F_{z_{m}, z_{m+1}}(\phi(t)) \geq F_{z_{m-1}, z_{m}}(t), \quad \forall t>0,
$$

i.e.,

$$
F_{z_{m}, z_{m+1}}(t) \geq F_{z_{m-1}, z_{m}}\left(\phi^{-1}(t)\right), \quad \forall t>0 .
$$

On the other hand, it follows from Lemma 1.11 that, for any $\lambda \in(0,1)$,

$$
\begin{aligned}
E_{\lambda, F}\left(z_{m}, z_{m+1}\right) & =\inf \left\{t>0, F_{z_{m}, z_{m+1}}(t)>1-\lambda\right\} \\
& \leq \inf \left\{\phi(\phi)^{-1}(t)>0, F_{z_{m-1}, z_{m}}\left(\phi^{-1}(t)\right)>1-\lambda\right\} \\
& \leq \phi\left(\inf \left\{\phi^{-1}(t)>0, F_{z_{m-1}, z_{m}}\left(\phi^{-1}(t)\right)>1-\lambda\right\}\right) \\
& =\phi\left(\inf \left\{t>0, F_{z_{m-1}, z_{m}}(t)>1-\lambda\right\}\right) \\
& =\phi\left(E_{\lambda, F}\left(z_{m-1}, z_{m}\right)\right) .
\end{aligned}
$$

By induction, we can prove that

$$
E_{\lambda, F}\left(z_{m}, z_{m+1}\right) \leq \phi\left(E_{\lambda, F}\left(z_{m-1}, z_{m}\right)\right) \leq \cdots \leq \phi^{m}\left(E_{\lambda, F}\left(z_{0}, z_{1}\right)\right) .
$$

By Lemma 1.7, for any given $\lambda \in(0,1)$ and for any positive integers $m, n, m>n$, there exists $\mu \in(0, \mu]$ such that

$$
\begin{aligned}
E_{\lambda, F}\left(z_{m}, z_{n}\right) & \leq E_{\mu, F}\left(z_{m}, z_{m-1}\right)+E_{\mu, F}\left(z_{m-1}, z_{m-2}\right)+\cdots+E_{\mu, F}\left(z_{n-1}, z_{n}\right) \\
& \leq \sum_{j=n}^{m-1} \phi^{j}\left(E_{\mu, F}\left(z_{0}, z_{1}\right)\right) \\
& \leq \sum_{j=n}^{m-1} \phi^{j}\left(E_{F}\left(z_{0}, z_{1}\right)\right) \rightarrow 0 \quad(\text { as } n, m \rightarrow \infty) .
\end{aligned}
$$


This implies that $\left\{z_{n}\right\}$ is a Cauchy sequence in $X$. Without loss of generality, we can assume that $z_{n} \rightarrow z^{*} \in X$. Therefore

$$
\begin{aligned}
f\left(x_{2 n}\right)=z_{2 n-1} \rightarrow z^{*} ; & g\left(x_{2 n+1}\right)=z_{2 n} \rightarrow z^{*} ; \\
S\left(x_{2 n}\right)=z_{2 n} \rightarrow z^{*} ; & G\left(x_{2 n+1}\right)=z_{2 n+1} \rightarrow z^{*} .
\end{aligned}
$$

By the assumption, without loss of generality, we can assume that $f$ is continuous, then $f^{2}\left(x_{2 n}\right) \rightarrow f\left(z^{*}\right)$ and $f S\left(x_{2 n}\right) \rightarrow f\left(z^{*}\right)$. Since $S$ and $f$ are compatible, we have

$$
F_{S f\left(x_{2 n}\right), f S\left(x_{2 n}\right)}(t) \rightarrow H(t), \quad \forall t>0,
$$

and so we have

$$
F_{S f\left(x_{2 n}\right), f z^{*}}(t) \geq T\left(F_{S f\left(x_{2 n}\right), f S\left(x_{2 n}\right)}(t-\phi(t))\right), \quad F_{f S\left(x_{2 n}\right), f z^{*}}(\phi(t)) \rightarrow H(t), \quad \forall t>0 .
$$

This shows that

$$
S f x_{2 n} \rightarrow f\left(z^{*}\right) \quad(\text { as } n \rightarrow \infty) .
$$

Again for any positive integer $n \geq 1$ and $\lambda \in(0,1)$, from Lemma 1.11, we have

$$
\begin{aligned}
E_{\lambda, F}\left(S f\left(x_{2 n}\right), G\left(x_{2 n+1}\right)\right)= & \inf \left\{t>0, F_{S f\left(x_{2 n}\right), G x_{2 n+1}}(t)>1-\lambda\right\} \\
\leq & \inf \left\{\phi(\phi)^{-1}(t)>0, \min \left\{F_{f^{2}\left(x_{2 n}\right), g\left(x_{2 n+1}\right)}\left(\phi^{-1}(t)\right),\right.\right. \\
& \left.\left.F_{f^{2}\left(x_{2 n}\right), S f\left(x_{2 n}\right)}\left(\phi^{-1}(t)\right), F_{g\left(x_{2 n+1}\right), G\left(x_{2 n+1}\right)}\left(\phi^{-1}(t)\right)\right\}\right\} \\
\leq & \phi\left(\operatorname { m a x } \left\{E_{\lambda, F}\left(f^{2}\left(x_{2 n}\right), g\left(x_{2 n+1}\right)\right), E_{\lambda, F}\left(f^{2}\left(x_{2 n}\right), S f\left(x_{2 n}\right)\right),\right.\right. \\
& \left.\left.E_{\lambda, F}\left(g\left(x_{2 n+1}\right), G\left(x_{2 n+1}\right)\right)\right\}\right) .
\end{aligned}
$$

Therefore we have

$$
\lim _{n \rightarrow \infty} E_{\lambda, F}\left(S f\left(x_{2 n}\right), G x_{2 n+1}\right)=E_{\lambda, F}\left(f z^{*}, z^{*}\right) \leq \phi\left(E_{\lambda, F}\left(f\left(z^{*}\right), z^{*}\right) .\right.
$$

By Remark $1.10, E_{\lambda, F}\left(f z^{*}, z^{*}\right)=0$, i.e., $z^{*}=f z^{*}$.

Similarly, we can prove that

$$
\begin{aligned}
E_{\lambda, F}\left(S z^{*}, G\left(x_{2 n+1}\right)\right) \leq & \phi\left(\operatorname { m a x } \left\{E_{\lambda, F}\left(\left(f z^{*}\right), g\left(x_{2 n+1}\right)\right), E_{\lambda, F}\left(f z^{*}, S z^{*}\right),\right.\right. \\
& \left.\left.E_{\lambda, F}\left(g\left(x_{2 n+1}\right), G\left(x_{2 n+1}\right)\right)\right\}\right) .
\end{aligned}
$$

Hence we have

$$
\begin{aligned}
E_{\lambda, F}\left(S z^{*}, z^{*}\right) & =\lim _{n \rightarrow \infty} E_{\lambda, F}\left(S z^{*}, G\left(x_{2 n+1}\right)\right) \\
& \leq \lim _{n \rightarrow \infty} \phi\left(\max \left\{E_{\lambda, F}\left(f z^{*}, g\left(x_{2 n+1}\right)\right), E_{\lambda, F}\left(f z^{*}, S z^{*}\right), E_{\lambda, F}\left(g\left(x_{2 n+1}\right), G\left(x_{2 n+1}\right)\right)\right\}\right) \\
& =\phi\left(\max \left\{0, E_{\lambda, F}\left(S z^{*}, z^{*}\right), 0\right\}\right) \\
& =\phi\left(E_{\lambda, F}\left(S z^{*}, z^{*}\right)\right)
\end{aligned}
$$

and so $E_{\lambda, F}\left(S z^{*}, z^{*}\right)=0$, i.e., $S z^{*}=z^{*}$. 
Select $z \in X$ such that $g(z)=z^{*}=S\left(z^{*}\right)$. Then $G(g(z))=G\left(z^{*}\right)$ and for any $\lambda \in(0,1)$

$$
\begin{aligned}
E_{\lambda, F}\left(z^{*}, G(z)\right)= & E_{\lambda, F}\left(S z^{*}, G(z)\right) \\
= & \inf \left\{t>0, F_{S z^{*}, G(z)}(t)>1-\lambda\right\} \\
\leq & \inf \left\{\phi(\phi)^{-1}(t)>0, \min \left\{F_{f\left(z^{*}\right), g(z)}\left(\phi^{-1}(t)\right), F_{f\left(z^{*}\right), S\left(z^{*}\right)}\left(\phi^{-1}(t)\right),\right.\right. \\
& \left.\left.F_{g(z), G(z)}\left(\phi^{-1}(t)\right)\right\}>1-\lambda\right\} \\
\leq & \phi\left(\max \left\{E_{\lambda, F}\left(f\left(z^{*}\right), g(z)\right), E_{\lambda, F}\left(f\left(z^{*}\right), S\left(z^{*}\right)\right), E_{\lambda, F}(g(z), G(z))\right\}\right) \\
= & \phi\left(\max \left\{E_{\lambda, F}\left(z^{*}, z^{*}\right), E_{\lambda, F}\left(z^{*}, S\left(z^{*}\right)\right), E_{\lambda, F}\left(z^{*}, G(z)\right)\right\}\right) \\
= & \phi\left(\max \left\{0,0, E_{\lambda, F}\left(z^{*}, G(z)\right)\right\}\right) .
\end{aligned}
$$

This implies that $z^{*}=G(z)$, and so $g(G(z))=g\left(z^{*}\right)$. Since $G, g$ are compatible and $E_{\lambda, F}(G(z), g(z))=E_{\lambda, F}\left(z^{*}, z^{*}\right)=0$, we get

$$
E_{\lambda, F}\left(G z^{*}, g\left(z^{*}\right)\right)=E_{\lambda, F}(G(g(z)), g(G(z)))=0 \text {. }
$$

This shows that $G z^{*}=g\left(z^{*}\right)$. Again for any $\lambda \in(0,1)$

$$
\begin{aligned}
E_{\lambda, F}\left(z^{*}, G\left(z^{*}\right)\right)= & E_{\lambda, F}\left(S\left(z^{*}\right), G\left(z^{*}\right)\right) \\
= & \inf \left\{t>0, F_{S\left(z^{*}\right), G\left(z^{*}\right)}(t)>1-\lambda\right\} \\
\leq & \inf \left\{\phi(\phi)^{-1}(t)>0, \min \left\{F_{f\left(z^{*}\right), g\left(z^{*}\right)}\left(\phi^{-1}(t)\right), F_{f\left(z^{*}\right), S\left(z^{*}\right)}\left(\phi^{-1}(t)\right),\right.\right. \\
& \left.\left.F_{g\left(z^{*}\right), G\left(z^{*}\right)}\left(\phi^{-1}(t)\right)\right\}>1-\lambda\right\} \\
\leq & \phi\left(\operatorname { i n f } \left\{\phi^{-1}(t)>0, \min \left\{F_{z^{*}, G\left(z^{*}\right)}\left(\phi^{-1}(t)\right), F_{z^{*}, z^{*}}\left(\phi^{-1}(t)\right),\right.\right.\right. \\
& \left.\left.\left.F_{G\left(z^{*}\right), G\left(z^{*}\right)}\left(\phi^{-1}(t)\right)\right\}>1-\lambda\right\}\right) \\
= & \phi\left(\max \left\{E_{\lambda, F}\left(z^{*}, G\left(z^{*}\right)\right), 0,0\right\}\right) \\
= & \phi\left(E_{\lambda, F}\left(z^{*}, G\left(z^{*}\right)\right) .\right.
\end{aligned}
$$

This implies that $E_{\lambda, F}\left(z^{*}, G\left(z^{*}\right)\right)=0$, i.e., $z^{*}=G\left(z^{*}\right)$, and so $z^{*}$ is a common fixed point of $f, S, g, G$ in $X$.

If $y \in X$ is also a common fixed point of $f, S, g, G$, then we have

$$
\begin{aligned}
E_{\lambda, F}\left(y, z^{*}\right)= & E_{\lambda, F}\left(S(y), G\left(z^{*}\right)\right) \\
= & \inf \left\{t>0, F_{S(y), G\left(z^{*}\right)}(t)>1-\lambda\right\} \\
\leq & \inf \left\{t>0, \min \left\{F_{y, z^{*}}\left(\phi^{-1}(t)\right), F_{f(y), S(y)}\left(\phi^{-1}(t)\right),\right.\right. \\
& \left.\left.F_{g\left(z^{*}\right), G\left(z^{*}\right)}\left(\phi^{-1}(t)\right)\right\}>1-\lambda\right\} \\
\leq & \phi\left(\max \left\{E_{\lambda, F}\left(y, z^{*}\right), 0,0\right\}\right) \\
= & \phi\left(E_{\lambda, F}\left(y, z^{*}\right)\right),
\end{aligned}
$$

i.e., $y=z^{*}$. This completes the proof of Theorem 3.3. 
Remark 3.4 If the mappings $S, G: X \rightarrow X$ given in Theorem 3.3 are multi-valued, we can also prove that the conclusion of Theorem 3.3 still holds, i.e., $y=z^{*}$. This completes the proof of Theorem 3.3.

\section{Competing interests}

The authors declare that they have no competing interests.

\section{Authors' contributions}

The authors contributed equally to the writing of the present article. And they also read and approved the final manuscript.

\section{Acknowledgements}

This study was supported by the National Natural Science Foundation of China (Grant No. 11361070). The authors would like to express their thanks to the reviewers for their helpful comments and suggestions.

Received: 24 February 2014 Accepted: 7 August 2014 Published: 04 Sep 2014

\section{References}

1. Schweizer, B, Sklar, A: Probabilistic Metric Spaces. Elsevier, New York (1983)

2. Chang, SS, Cho, YJ, Kang, SM: Nonlinear Operator Theory in Probabilistic Metric Spaces. Nova Science Publishers, New York (2001)

3. Alsina, C, Schweizer, B, Sklar, A: Continuity properties of probabilistic norms. J. Math. Anal. Appl. 208, 446-452 (1997)

4. Bharucha-Reid, A: Fixed point theorems in probabilistic analysis. Bull. Am. Math. Soc. 82, $641-657$ (1976)

5. Chang, SS, Lee, BS, Cho, YJ, Chen, YQ: Generalized contraction mapping principle and differential equations in probabilistic metric spaces. Proc. Am. Math. Soc. 124(8), 641-657 (1996)

6. Hadžić, O, Pap, E: Fixed Point Theory in PM Spaces. Kluwer Academic, Dordrecht (2001)

7. Chang, SS: Fixed Point Theory and Applications. Chongqing Publishing Press, Chongqing (1984) (in Chinese)

8. $\mathrm{Hu}, \mathrm{X}-\mathrm{q}, \mathrm{Ma}, \mathrm{X}-\mathrm{y}$ : Coupled coincidence point theorems under contractive conditions in partially ordered probabilistic metric spaces. Nonlinear Anal. (2011). doi:10.1016/j.na.2011.06.028

9. O'Regan, D, Saadati, R: Nonlinear contraction theorems in probabilistic spaces. Appl. Math. Comput. 195, 86-93 (2008)

10. Jungck, G: Commuting maps and fixed points. Am. Math. Mon. 83, 261-263 (1976)

11. Singh, B, Jain, S: A fixed point theorem in Menger space through weak compatibility. J. Math. Anal. Appl. 301, 439-448 (2005)

10.1186/1029-242X-2014-347

Cite this article as: Tang et al.: Nonlinear contractive and nonlinear compatible type mappings in Menger probabilistic metric spaces. Journal of Inequalities and Applications 2014, 2014:347

\section{Submit your manuscript to a SpringerOpen ${ }^{\circ}$ journal and benefit from:}

- Convenient online submission

- Rigorous peer review

- Immediate publication on acceptance

- Open access: articles freely available online

- High visibility within the field

- Retaining the copyright to your article 